\title{
Der Peinlichkeit entkommen
}

Rolf Bossart

Nach der Präsentation des neuen Essaybandes meines Freundes sassen wir zusammen und machten Witze über den lilafarbenen Umschlag, den der Verlag dem Buch gegeben hatte. Eine Mitarbeiterin unseres Instituts machte mit meinem Handy ein feuchtfröhliches Foto von den "Lilaboys«. Wieder zu Hause wollte ich ihr dieses Foto schicken mit »lieben Grüssen von den Lilaboys". Niemand weiss, wieso es nicht an sie, sondern an eine Schülerin der Maturaklassen ging, deren Nummer nur deshalb auf meinem Handy war, weil sie mir vor einigen Tagen per Whats App ein Thesenpapier geschickt hatte. Als ich die Fehlsendung bemerkte, war es zu spät. Die Schülerin hatte das Bild ohne Rückfrage in den Klassenchat gestellt. Eine aufklärende Nachricht blieb unbeantwortet. Am nächsten Morgen erklärte ich das Missgeschick einigen Schülern. Wir lachten gerade über die Sache, als der Rektor mich in sein Büro bat und mir mitteilte, dass sich vorige Nacht ein Kollege das Leben genommen hatte.

Aus der Perspektive der Peinlichkeit betrachtet, erzählt sich diese Geschichte etwa so: Dieses Foto mit der Widmung der Mitarbeiterin zu schicken, schien für mich logisch, denn sie hatte es ja gemacht. Doch schon meinem Freund wollte ich es nicht senden, da dies nicht sein Stil war. Eine allfällige abwertende Reaktion von ihm wäre mir peinlich gewesen. Entsprechend peinlich war mir die Fehlsendung an die Schülerin, aber doch auch nicht wieder sehr peinlich, da es sich um eine Schülerin handelte, zu der ich sehr neutral, also weder besonders positiv, noch besonders negativ in Beziehung stand. (Würde ich nun aber denken, dass sie diese Zeilen liest, wäre mir diese Aussage peinlich, und ich würde versuchen, das Verhältnis etwas netter zu formulieren.) Als ich annehmen musste, dass die halbe Schule dieses Foto bekommen hatte, war mir das natürlich peinlich, vor allem wegen der albernen Widmung, aber auch wieder nicht so peinlich, nicht nur, weil ich wusste, dass die Schüler mir grundsätzlich wohlwollend gegenüber stehen, sondern vor allem auch, weil ich wusste, wie viele solcher Fotos heute verschickt werden und dass ein solches Ereignis sehr schnell wieder vergessen wird. Allerdings war mir Letzteres dann aus anderer Perspektive betrachtet wiederum peinlich, da ich ja eigentlich gerade nicht zu jenen »Föteliverschickern« gehören möchte. 
Und die Nachricht von der Selbsttötung meines Kollegen? Was bewirkte sie? Die ganzen Peinlichkeiten wichen der Scham, mit den falschen Dingen beschäftigt zu sein, und dem Gefühl, an dieser Tragödie irgendwie schuld zu sein. Doch später, nachdem die Schuldgefühle einigermassen verarbeitet und geordnet waren, meldete sich bereits wieder die Peinlichkeit. Es war mir unangenehm, dass ich damals das Missgeschick nicht genug breit aufklären konnte und ich also bis heute damit rechnen muss, dass einige glauben, ich hätte sowas absichtlich an Schüler verschickt. Aber nach allem war es mir natürlich ebenso zu peinlich, nochmal darauf zurückzukommen, und ich unterliess es.

Warum aber ist dieses Wechselbad der Peinlichkeiten nicht einfach nur unangenehm für den Einzelnen, sondern auch gesellschaftlich eine höchst unbefriedigende Angelegenheit und Zeitverschwendung? Ich möchte versuchen, im Folgenden diese Frage etwas zu erörtern.

Die höchst bemerkenswerte Ausgangslage dieser Geschichte ist die, dass ich nichts getan hatte, was ich hatte tun wollen und trotzdem hätte daran schuld sein sollen. Ich hatte weder etwas versucht, noch etwas gewagt. Es war mir einfach passiert. Ich war, um einen modernen Rechtsbegriff zu verwenden, einfach nur fahrlässig.

Das Fahrlässige ist in der technisierten Welt, in der wir leben, eine wichtige Kategorien des Handelns geworden. Die Fahrlässigkeit ist weder richtig fremd verschuldet noch eigen verschuldet. Sie befindet sich in einem seltsamen Zwischenraum, in dem die alte Dichotomie von Schicksal und Autonomie beziehungsweise Freiheit des Handelns nicht zählt. Das heisst, schuld an den Folgen der Fahrlässigkeit sind weder höhere Mächte noch andere Menschen, noch eigentlich ich selber.

Wir leben in einer Situation, in der es kaum mehr Dinge gibt, die nicht irgendwie auf menschliche Einwirkungen zurückgehen, wobei aber ein immer grösserer Teil dieser Ereignisse nicht direkt einem menschlichen Willen entspringt, sondern Verkettungen mit einem Auslöser, dem entweder keine bestimmte Absicht oder keine Abschätzung der Folgen unterstellt werden kann.

Das Recht regelt zwar die Verantwortlichkeiten. Es unterscheidet zwischen Fahrlässigkeit und Zufall. Ein Zufall liegt im Zivilrecht dann vor, wenn für ein Ereignis kein Vorsatz nachweisbar ist. Aber das ist noch nicht alles. Es darf auch keine Fahrlässigkeit vorhanden sein. Das bedeutet, wenn mein Auto die Strasse runterrollt, ist es nur ein Zufall, wenn ich vorher die Handbremse angezogen habe und wenn diese intakt ist. Was aber liegt vor, wenn ich beobachte, wie 
ein Blumentopf vom vierten Stock herunterfällt und ich es unterlasse, den Passanten, der in diesem Moment des Weges kommt, zu warnen? Das heisst, in Tat und Wahrheit gibt es unzählige Fälle, bei denen man irgendwie weiss, wer beteiligt war, aber die UrsacheWirkung-Zusammenhänge nicht klar ersichtlich sind und auch nicht klar nachgewiesen werden können.

Die Kategorie der Peinlichkeit gehört nun in dieses Feld der Wirkungen ohne bestimmte Absicht. Sie ist das Resultat einer spezifischen Untätigkeit. Auch wenn die modernen Gesellschaften viele Gelegenheiten bereitstellen zu dieser Untätigkeit, so ist es heute doch vor allem die digitale Welt, die uns dazu verleitet. Und daher möchte ich daran die gesellschaftliche Problematik des Peinlichen etwas ausführen. Klicken, liken, followen. Senden, teilen, weiterleiten. Ansehen, kopieren, kommentieren. Dies sind die narzisstischen Untaten der Web-Community. Was die Web-Community tut, tut sie für jedermann. Aber jedermann ist, wenn's drauf ankommt, keiner. Was man für jedermann tut, tut man daher in gewisser Weise nur für sich allein. Wo weder Absender noch Adressat ersichtlich sind, ist der User auf sich selbst geworfen, er ist mit sich allein. Das Ich, sofern es die Funktion der Selbststeuerung in Relation zu den Anforderungen der Aussenwelt hatte, verliert seine Bedeutung. Denn die Aussenwelt wird nur als Auge halluziniert, das einem so sieht, wie man es gerne hätte, die einem also mit allem, was man tut, ins Recht setzen soll. Und da die Aussenwelt im World Wide Web nicht mehr als verbindliches Korrektiv zur eigenen Triebstruktur erfahren wird, nicht mehr als Ort, der einem dazu auffordert, sich besser zu zeigen als man ist, ist es der Ort der Regression, der Ort, wo Es herrscht anstatt Ich.Von dem, was der User tut, kann man daher mit Recht sagen: Es passiert. Das World Wide Web ist in Bezug auf das Subjekt des Users die Antithese zur regulativen Öffentlichkeit: Es herrscht darin - um einmal mal mehr den treffenden Titel von Richard Sennett - zu zitieren - »die Tyrannei der Intimität» oder anders gesagt: ein Totalitarismus der Befindlichkeit.

Die Peinlichkeit tritt nun entweder dort auf, wo der User seine Netzuntaten plötzlich von realen Subjekten reflektiert sieht (wie in meinem Beispiel des an eine falsche Adresse gesendeten Fotos) oder wo die Web-Community sich über einen einzelnen User hermacht und ihn aus heiterem Himmel als Subjekt identifiziert und zur Rechenschaft zwingt wie im Fall des Shitstorms. Das heisst, Peinlichkeit stellt sich da ein, wo die eigentlich nicht mehr vorhandene Öffentlichkeit plötzlich als deren Phantom auftritt. Die Peinlichkeit ist der Phantomschmerz für die Scham. Denn Scham kann man nur 
haben, wo ein Akt vorliegt, eine Entscheidung, die man vor dem Tribunal der Öffentlichkeit zu rechtfertigen hat. Die Scham ist das Gefühl des Ungenügens einer Handlung gegenüber einer abstrakten Öffentlichkeit mit klaren, transparenten Forderungen. Sie fordert auf zur Erkenntnis von Gut und Böse und zur Entscheidung: Anpassung oder Widerstand. Die Peinlichkeit dagegen ist das Gefühl des Ungenügens des Soseins gegenüber einer Community mit unklaren und intransparenten Forderungen. Sie stellt einen vor keine Wahl; es gibt nichts, was man tun könnte, weil man missfällt, ohne dass man etwas Bestimmtes getan hätte. Die Scham ist zu bearbeiten vom Individuum. Es kann, um sie los zu werden, beschliessen, sich zu ändern oder für die Veränderung der Gesellschaft zu kämpfen. Die Peinlichkeit ist bloss hinzunehmen.

Bei Nietzsche ist es das Charakteristikum der hüpfenden und blinzelnden Spottgestalt, die er den "Letzten Menschen" nennt, dass er sich nicht einmal mehr schämen kann. Denn er ist nicht mehr fähig, ein Ziel zu haben beziehungsweise eine Perspektive zu imaginieren, die über das, was das Ich hier und jetzt beschäftigt, hinausgeht. Die Peinlichkeit kann man im Anschluss daran bestimmen als Symptom einer Gesellschaft, die immer weniger durch eine abstrakte Öffentlichkeit dirigiert wird und immer mehr in Gruppen und Banden zerfallt wie die Worldweb-Community, die Konsumenten, die Mafia, der Bund der Steuerzahler oder die Bürgerwehr usw. Man kann sagen: je weniger Öffentlichkeit, desto mehr Peinlichkeit. Und wie der Wortstamm schon vermuten lässt, ist der Schritt von der harmlosen Peinlichkeit zur Pein letztlich nur sehr klein.

Ich möchte das kurz an der Situation der Homosexuellen in europäischen Gesellschaften skizzieren. Die Homosexuellen-Bewegung kann beachtliche Erfolge ausweisen in der Reduktion rechtlicher Diskriminierung. Bei der alltäglichen Schwulenfeindlichkeit und der Verfolgung von Homosexuellen sieht es immer noch oder je nach Land wieder zunehmend düster aus. Das kann zum Paradox führen, das zum Beispiel Heiraten für Schwule vielerorts möglich und normal, aber Händchenhalten oder Küssen in der Öffentlichkeit wieder vermehrt ein Akt der Peinlichkeit ist. Nehmen wir als vergleichsweise harmloses Beispiel einen Artikel in der Süddeutschen Zeitung mit der Überschrift: "Nur die Mutigen halten Händchen «", der das Verschwinden der Schwulenszene aus ihrem angestammten Münchner Quartier und eine diffuse Schwulenfeindlichkeit der dort neu sich installierenden "Hetero-Shoppingszene" beschreibt.

\footnotetext{
1 Mathias Weber, Schwule Szene München. Nur die Mutigen halten Händchen, in: Süddeutsche Zeitung, 7.10.2014.
} 
Die rechtliche Gleichstellung der Homosexuellen hat in einer Gesellschaft, die sich als Reaktion auf die verschärften Krisen der Moderne wieder mehr als Gemeinschaft von verschiedenen Gruppen inszeniert und weniger über eine abstrakte, rechtlich bestimmte Öffentlichkeit definiert, keine grosse emanzipatorische Wirkung. Zum Beispiel sind die Schwulen in diesem Zusammenhang eine geeignete Projektionsfläche für den Hass auf die urbanen Siegertypen des Kapitalismus. Weil sie, so das Vorurteil, wegen ihrer "Ungebundenheit« und "Anpassungsfähigkeit« erfolgreich seien und die brüchigen Werte noch weiter untergraben würden. Das öffentliche Küssen der Homosexuellen ist angesichts solcher Ressentiments nicht ein Akt innerhalb eines Rechtsverhältnisses, der entweder verboten ist oder erlaubt, sondern er ist das Zeichen bzw. die Untat eines Soseins, woraus immer ein Moment der Peinlichkeit droht, je nach Gruppe, der man gerade gegenüber steht. Zu rechtfertigen hat man nicht eine Tat vor dem Gesetz, sondern sein Sosein vor den informellen Normen jeder beliebigen Gruppe oder Bande, die gerade den öffentlichen Raum besetzt.

Das Verschwinden der Scham ist in den letzten Jahrzehnten verschiedentlich beklagt worden. Wenn ich nun hier versucht habe, die Peinlichkeit als ihr postmodernes Surrogat zu skizzieren, dann nicht, um die Scham als unproblematisch auszuweisen, sondern um zu zeigen, dass ihre Problematik sich nicht erledigt hat. Das Aushalten der eigenen Peinlichkeit und die kollektive Belustigung an der Peinlichkeit des Einzelnen durch Gruppen kann natürlich harmloser, ja befreiender sein als das öffentliche Schämen. Die Peinlichkeit kann aber auch genauso gut kippen in Formen der totalen Ohnmacht des Einzelnen beziehungsweise kann sie der Ermutigung zur Selbstjustiz von Gruppen und Banden dienen. Sie ist auf jeden Fall aber das Symptom einer Handlungsarmut. Wo viel Peinlichkeit, da ist wenig Handlungsmöglichkeit. Der Diskurs der Peinlichkeit ist der Spottvers auf eine höchst heterogene, aber im Wesentlichen alternativlose Gesellschaft.

Doch was kann man unter den aktuellen Bedingungen dem Diskurs der Peinlichkeit entgegensetzen? Was kann man den Individuen raten, die sich ständig neuen Community-Anforderungen gegenüber sehen und deren Energien damit erschöpft werden, sich permanent neu dazu zu verhalten? Um die Handlungspotenz des Einzelnen zu stärken, wäre es notwendig, aus dem Gefängnis des grossen Auges der Anderen herauszutreten. Drei Punkte scheinen mir nützlich, um dem Diskurs der Peinlichkeit zu entkommen. Und es zeigt sich in allen drei Punkten, dass man dabei wohl der Pein- 
lichkeit der Menschen, aber nicht der Pein im Sinne von Mühe des Menschseins entkommt, in Form der Entsagung, der Leidenschaft und der Enttäuschung.

1. Nicht alles tun: Nicht alles tun, heisst heute, den tausendfältigen Möglichkeiten der Nicht- und Untaten zu entsagen. Je weniger man macht, das man eigentlich gar nicht tun wollte und das mangels konkreten Adressaten nur eine "narzisstische Untat« genannt werden kann, je weniger man also liked, hated, Föteli postet, kommentiert, an seinem Outfit bastelt usw., desto weniger muss man sich identifizieren und desto weniger läuft man Gefahr, von anderen damit identifiziert und der Peinlichkeit überführt zu werden.

2. Mut zur Lächerlichkeit: Das Einzige, was sich wirklich lohnt zu tun, ist das, was man mit Leidenschaft tun kann. Die »Pein« der Tat nimmt die Pein der Peinlichkeit vorweg. Es ist das Wesen der Leidenschaft, dass man sich zeigt und kenntlich macht, ungeschützt und naiv. Aber was dabei belächelt und verspottet werden kann, ist paradoxerweise nicht das Sosein, sondern die Art und Weise des Handelns, das Handeln selbst oder das Werk. Die Leidenschaft ist die Wette darauf, dass man wirklich irgendwo gebraucht wird.

3. Fröhlich scheitern: Alles, was man mit Leidenschaft tut, kann scheitern. Das Meiste, das man auf dieser Welt überhaupt mit Leidenschaft tun kann, ist zum Scheitern verurteilt, weil es sich dabei um die unlösbaren und schwierigen Dinge handelt. Wer fröhlich scheitert, weiss um die prinzipielle Unlösbarkeit der gesetzten Aufgabe, er braucht sie sich nicht erst vorführen zu lassen. Aber das fröhliche Scheitern unterscheidet sich von den Vermeidungsstrategien des Zynikers vor Enttäuschungen gerade dadurch, dass man das Leiden an der Enttäuschung nicht umgeht, sondern vielmehr weiss, dass man nur weiter kommt, wenn man immer von Neuem enttäuschbar bleibt. Das heisst, dass man sich immer wieder von Neuem den Illusionen der Veränderbarkeit der Zustände hingibt und sich nicht entmutigen lässt durch Enttäuschung.

- Dr. theol. Rolf Bossart ist Lehrer für Religionswissenschaften, Psychologie und Pädagogik, Redaktor bei theoriekritik.ch und Mitarbeiter in "theorie design" beim International Institute of Political Murder (IIPM). 